\title{
Clinical evaluation of rosuvastatin in heart transplant patients with hypercholesterolemia and therapeutic failure of other statin regimens: short-term and long-term efficacy and safety results
}

\author{
Gonzalo Barge-Caballero, ${ }^{1,2}$ Eduardo Barge-Caballero, ${ }^{1,2}$ Raquel Marzoa-Rivas, ${ }^{1,2}$ María \\ J. Paniagua-Martín, ${ }^{1,2}$ Alfredo Barrio-Rodríguez, ${ }^{1,2}$ Carmen Naya-Leira, ${ }_{1,2}$ Paula Blanco- \\ Canosa, ${ }^{1,2}$ Zulaika Grille-Cancela, ${ }^{1,2}$ José Manuel Vázquez-Rodríguez, ${ }^{1,2}$ and María G. \\ Crespo-Leiro, ${ }^{1,2}$
}

\footnotetext{
${ }^{1}$ Servicio de Cardiología, Complejo Hospitalario Universitario de A Coruña, A Coruña, Spain

${ }^{2}$ Instituto de Investigación Biomédica de A Coruña (INIBIC), A Coruña, Spain
}

\begin{abstract}
Summary
We conducted an observational study of 30 heart transplant recipients with serum low-density lipoprotein cholesterol (LDL-c) $>100 \mathrm{mg} / \mathrm{dl}$ despite previous statin therapy, who were treated with rosuvastatin $10 \mathrm{mg}$ daily $(5 \mathrm{mg}$ in case of renal dysfunction). Serum lipids, creatine phosphokinase (CPK), bilirubin, and hepatic enzymes were prospectively measured 2, 4, and 12 weeks after the initiation of the drug. Clinical outcomes of patients who continued on longterm rosuvastatin therapy beyond this 12-week period were reviewed in February 2015. Over the 12-week period following rosuvastatin initiation, serum levels of total cholesterol (TC) and LDL-c and the ratio TC/high-density lipoprotein cholesterol (HDL-c) decreased steadily $(P<0.001)$. Average absolute reductions of these three parameters were $-48.7 \mathrm{mg} / \mathrm{dl},-46.6 \mathrm{mg} / \mathrm{dl}$, and -0.9 , respectively. Seventeen $(57 \%)$ achieved a serum LDLc $<100 \mathrm{mg} / \mathrm{dl}$. No significant changes from baseline were observed in serum levels of triglycerides, HDL-c, hepatic enzymes, bilirubin, or CPK. Twenty-seven (90\%) patients continued on long-term therapy with rosuvastatin over a median period of 3.6 years, with no further significant variation in lipid profile. The drug was suspended due to liver toxicity in $1(3.3 \%)$ patient and due to muscle toxicity in $2(6.7 \%)$ patients. All adverse reactions resolved rapidly after rosuvastatin withdrawal. Our study supports rosuvastatin as a reasonable alternative for heart transplant recipients with hypercholesterolemia and therapeutic failure of other statin regimens.
\end{abstract}

\section{Keywords}

Heart transplantation; Hypercholesterolemia; Rosuvastatin. 


\section{Introduction}

Statins are cholesterol-lowering agents that inhibit the endogenous synthesis of cholesterol by blocking the hydroxymethylglutaryl-CoA reductase. In patients with previous history of coronary artery disease and in those with high cardiovascular risk, chronic treatment with statins has shown a significant reduction in serum levels of total cholesterol (TC) and low-density lipoprotein cholesterol (LDL-c), as well as a decreased incidence of cardiovascular events [1,2].

Hypercholesterolemia is a common comorbidity in patients with heart transplantation (HT), with a prevalence reaching 60-80\% [3, 4]. Hypercholesterolemia may occur as a pre-existing condition, but also as a side effect of immunosuppressive therapy [5], leading to an increased risk of cardiovascular disease and mortality, as well as to the development of coronary allograft vasculopathy [6]. In HT patients, statin therapy has shown a significant reduction in the incidence of acute rejection and CAV and increased long-term survival [7, 8]. However, the use of statins in this population is conditioned by its potential interactions with immunosuppressive therapy and an increased risk of adverse events $[9,10]$.

Rosuvastatin is a synthetic statin [11] with high lipid-lowering power and a suitable safety profile derived from its hydrophilic nature. So far, only a small observational study has evaluated its clinical usefulness in patients with HT. In this previous report [12], rosuvastatin showed good efficacy and a low rate of serious adverse events over limited follow-up of 6 weeks. To the best of our knowledge, however, no long-term clinical data about the clinical usefulness of this drug in the HT population have been reported until now.

The aim of our study was to assess the efficacy and safety of rosuvastatin therapy in HT patients with uncontrolled hypercholesterolemia despite previous treatment with other statin regimens, both in the short term and also in the long term.

\section{Methods}

\section{Study protocol}

We conducted a prospective observational study between September 2009 and March 2010. A total of 30 patients with HT who had a serum LDL-c $>100 \mathrm{mg} / \mathrm{dl}$ despite statin therapy at the highest tolerated dose or in which statins had been discontinued indefinitely due to adverse reactions were included. Concomitant treatment with other lipid-lowering agents, such as ezetimibe, was considered an exclusion criterion for the study.

After discontinuing previous treatment, patients initiated rosuvastatin $10 \mathrm{mg} /$ day in a single oral nightly dose. Starting dose was reduced to $5 \mathrm{mg}$ in patients who showed a serum creatinine $>1.5 \mathrm{mg} / \mathrm{dl}$ at baseline, allowing dose escalation to $10 \mathrm{mg} /$ day after four weeks, only if they showed good tolerance, no adverse effects and serum LDL-c remained above $100 \mathrm{mg} / \mathrm{dl}$.

All patients received immunosuppressive treatment with varying doses of oral prednisone depending on the time passed after HT and the number of previous episodes of graft rejection. Patients also received a combination of at least two of the following three drug groups: (i) calcineurin inhibitors (cyclosporine or tacrolimus), (ii) mTOR inhibitors (everolimus or sirolimus), and (iii) mycophenolate mofetil or sodium mycophenolate.

According to a predefined protocol, scheduled clinical visits were conducted at 2, 4, and 12 weeks after initiation of treatment. At every visit, analytical tests were performed, including the determination of plasma levels of TC, triglycerides, LDL-c, HDL-c, bilirubin, creatinine phosphokinase (CPK), glutamate oxaloacetate transaminase (GOT), and glutamate pyruvate transaminase (GPT). Patients were enquired about tolerability and potential side effects of the study drug. Patients who showed an LDL-c $<100 \mathrm{mg} / \mathrm{dl}$ at the end of this 12-week follow-up period were considered as "responders" to rosuvastatin therapy. Secondary therapeutic goals were a serum TC $<200 \mathrm{mg} / \mathrm{dl}$, serum triglyceride $<150 \mathrm{mg} / \mathrm{dl}$, and a TC/HDL-c ratio $<4$. 
In February 2015, we conducted a retrospective review of long-term clinical outcomes of patients who continued on rosuvastatin therapy beyond the predefined 12-week follow-up period on the basis of the clinical criterion of their attending cardiologist. The occurrence of drug-related adverse reactions and cardiovascular events (coronary and cerebrovascular) over long-term follow-up was recorded. Laboratory data at the time of the last clinical visit were also collected.

\section{Definitions}

Liver toxicity was defined as the elevation of serum GPT and/or GOT >3 times the upper limit of standard reference values [12]. Episodes of muscle toxicity were classified according to the Canadian Working Group [13] definitions:

1. Myalgia: muscle symptoms with normal or mildly elevated serum CPK levels.

2. Mild CPK elevation: asymptomatic elevation of serum CPK levels up to 10 times the upper limit of normality.

3. Rhabdomyolysis: muscle symptoms and serum CPK levels $>10$ times the upper limit of normality (or $>10000 \mathrm{IU} / \mathrm{l})$, myoglobinuria, renal failure, or need for hydration.

The comparison of the daily doses of statins that patients were receiving before entering the study was conducted through the conversion to pravastatin equivalents, by applying the following conversion ratio: $40 \mathrm{mg}$ pravastatin $=10 \mathrm{mg}$ atorvastatin $=80 \mathrm{mg}$ fluvastatin .

\section{Statistical analysis}

In this study, categorical variables are presented as proportions and continuous variables are presented as median (interquartile range, IQR), due to a small sample size and asymmetrical distributions.

The trend of the serum levels of TC, LDL-c, HDL-c, triglycerides, TC/HDL-c ratio, GOT, GPT, and CPK over the predefined 12-week follow-up period is depicted using box-plot graphs, and statistical comparison was conducted using the nonparametric Friedman test for multiple repeated measures. To estimate the absolute and relative mean reductions in serum total cholesterol, LDL-c, triglycerides, and TC/HDL-c ratio after 12 weeks of treatment as compared to baseline, we first computed these differences for each of the patients and then we subsequently calculated the mean and standard deviation (SD) of these distributions. Statistical analysis was performed by means of IBM SPSS Statistics for Macintosh, Version 20.0 (IBM Corp, Armonk, NY, USA) and epidat 4.1 (Consellería de Sanidade, Xunta de Galicia, Spain). Statistical significance was set as a $P$ value $<0.05$.

\section{Results}

\section{Baseline clinical characteristics}

Of the 30 patients studied, 4 (13\%) were women. The median age of the sample was 60.2 years (IQR: 53.3-67.4) and the median time elapsed since HT was 4.2 years (IQR: 1.3-9.4).

At baseline, median TC was $236 \mathrm{mg} / \mathrm{dl}$ (IQR: 214-264), median triglycerides were $159 \mathrm{mg} / \mathrm{dl}$ (IQR: 118-249), median LDL-c was $142 \mathrm{mg} / \mathrm{dl}$ (IQR: 125-162), median HDL-c was $56 \mathrm{mg} / \mathrm{dl}$ (IQR: 43-69), and median TC/HDL-c ratio was 4.5 (IQR: 3.7-5.3). According to inclusion criteria, all patients presented LDL-c > $100 \mathrm{mg} / \mathrm{dl}$. In addition, 27 patients $(90 \%)$ presented TC> $200 \mathrm{mg} / \mathrm{dl}, 17(57 \%)$ had triglycerides $>150 \mathrm{mg} / \mathrm{dl}$, and $11(63 \%)$ had a TC/HDL-c ratio $>4$.

Just before entering the study, $26(87 \%)$ patients were being treated with statins at maximum tolerated doses (14 pravastatin, 11 atorvastatin, and 1 fluvastatin). The mean equivalent daily dose of pravastatin was $36 \mathrm{mg}$ (IQR: 20-40 mg). Four patients (13\%) had received statins in the past, but they had been suspended indefinitely due to adverse reactions (3 cases of liver toxicity and 1 case of muscular toxicity). Table 1 shows the baseline characteristics of the study population. 
Table 1. Clinical characteristics of study participants

\begin{tabular}{lc}
\hline & \\
Age (years), median (IQR) & $60.2(53.3-67.4)$ \\
Female $(n, \%)$ & $4(15 \%)$ \\
Diabetes mellitus & $6(20 \%)$ \\
Hypertension & $20(67 \%)$ \\
Immunosuppressive therapy & \\
Cyclosporine & $9(30 \%)$ \\
Tacrolimus & $15(50 \%)$ \\
Sirolimus or everolimus & $13(43 \%)$ \\
Mycophenolate mofetil & $21(70 \%)$ \\
Prednisone & $30(100 \%)$ \\
Current treatment with statins & $26(87 \%)$ \\
Pravastatin & $11(37 \%)$ \\
Atorvastatin & $9(33 \%)$ \\
Fluvastatin & $1(3 \%)$ \\
Pravastatin equivalent daily dose (mg), median (IQR) & $36(20-40)$ \\
Prior statin toxicity & \\
Hepatotoxicity & $3(10 \%)$ \\
Muscle toxicity & $1(3 \%)$ \\
Body mass index (kg/m²), median (IQR) & $27.4(25.4-30.6)$ \\
Time since HT (years), median (IQR) & $4.2(1.3-9.4)$ \\
Baseline laboratory parameters, median (IQR) & \\
Total cholesterol (mg/dl) & $236(214-264)$ \\
LDL cholesterol (mg/dl) & $142(125-162)$ \\
HDL cholesterol (mg/dl) & $56(43-69)$ \\
Triglycerides (mg/dl) & $159(118-249)$ \\
Creatinine (mg/dl) & $1.35(1.15-1.65)$ \\
Total bilirubin (mg/dl) & $0.6(0.3-0.8)$ \\
GOT (IU/l) & $21(18-33)$ \\
GPT (IU/l) & $25(17-33)$ \\
CPK (IU/l) & $92(51-155)$ \\
& \\
\hline & \\
& \\
& \\
& \\
&
\end{tabular}

CPK, creatine phosphokinase; GOT, glutamate oxaloacetate transaminase; GPT, glutamate pyruvate transaminase, IQR, interquartile range; HT, heart transplantation.

Rosuvastatin was initiated at the standard dose of $10 \mathrm{mg} /$ day in $20(67 \%)$ patients, while the reduced dose of $5 \mathrm{mg} /$ day was initiated in $10(33 \%)$ patients who had baseline serum creatinine $>1.5 \mathrm{mg} / \mathrm{dl}$. In the latter group, the dose of rosuvastatin was increased in 6 patients to $10 \mathrm{mg} /$ day after 4 weeks of treatment with the initial dose, due to the persistence of serum LDL-c > $100 \mathrm{mg} / \mathrm{dl}$.

\section{Changes in lipid profile}

Over the predefined 12-week follow-up period, statistically significant decreasing trends in TC $(P<0.001)$, LDL-c $(P<0.001)$, and TC/HDL-c ratio $(P<0.001)$ were observed (Fig. 1$)$. As compared to baseline, rosuvastatin therapy resulted in an average absolute reduction of $-48.7 \mathrm{mg} / \mathrm{dl}$ (confidence interval (CI) 95\%: -34.2 to -63.1 ) in TC and $-46.6 \mathrm{mg} / \mathrm{dl}$ (CI 95\% -33.5 to -59.7 ) in LDL-c, which represented an average relative reduction in these parameters of $-20.1 \%$ (CI 95\% $-14.7 \%$ to $-25.5 \%$ ) and $-19.2 \%$ (CI 95\% $-14.6 \%$ to $-23.8 \%$ ), respectively. The TC/HDL-c ratio experienced an average absolute reduction of -0.9 (CI 95\% -0.5 to -1.3 ), although no significant variation in HDL-c $(P=0.99)$ was detected. An average absolute reduction of $-16.1 \mathrm{mg} / \mathrm{dl}$ (CI $95 \%-40.3$ to +8.3$)$ in triglycerides was also observed, but the decreasing trend of this parameter over time did not reach statistical significance 
$(P=0.16)$. At the end of this period, 18 patients $(60 \%)$ showed serum TC $<200 \mathrm{mg} / \mathrm{dl}, 17$ patients $(57 \%)$ showed serum LDL-c < $100 \mathrm{mg} / \mathrm{dl}$, and $21(70 \%)$ patients showed a TC/HDL-c ratio <4. Only 14 patients $(47 \%)$ presented serum triglycerides $<150 \mathrm{mg} / \mathrm{dl}$.
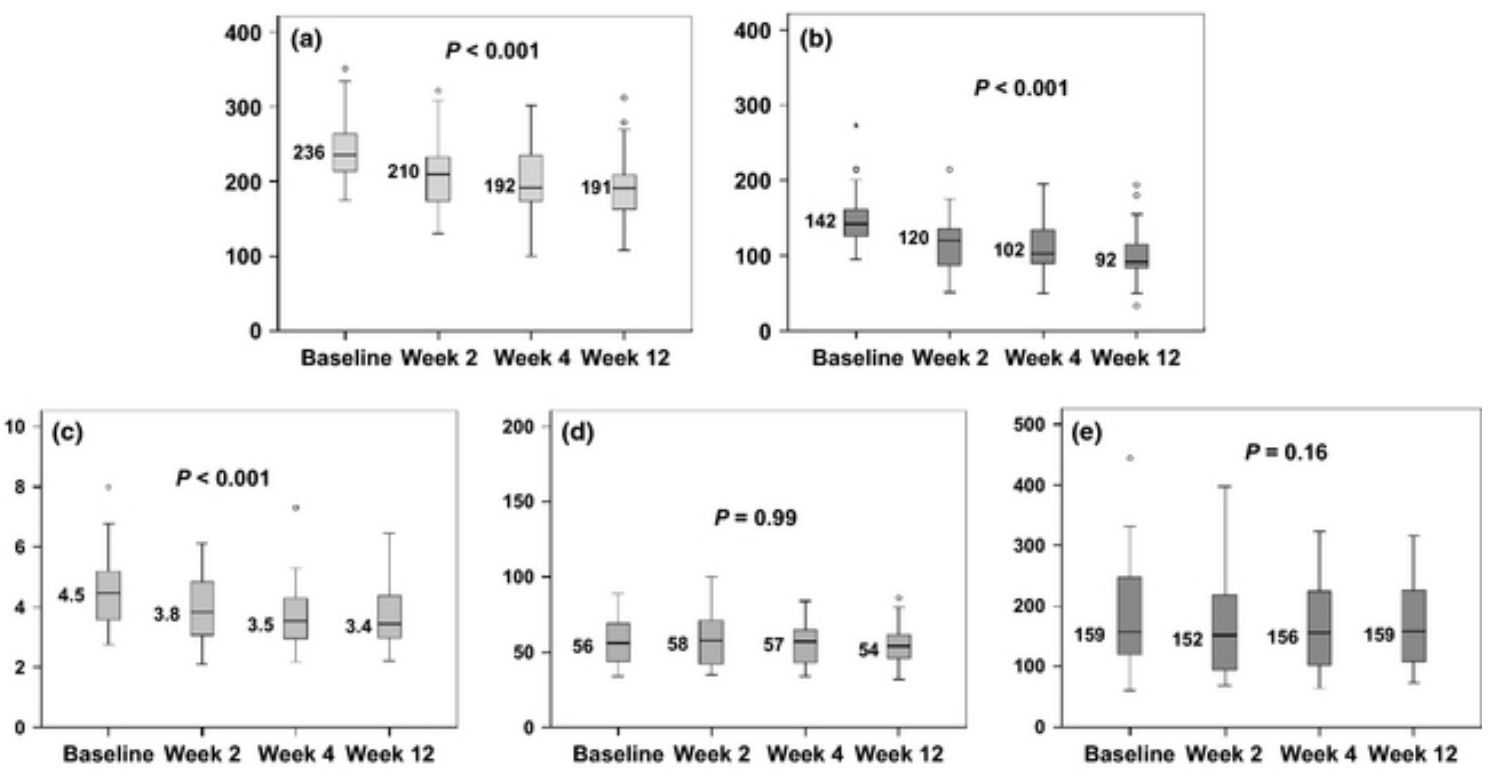

Figure 1. Evolution of serum total cholesterol (mg/dl, panel a), LDL cholesterol (mg/dl, panel b), total cholesterol total/HDL ratio (panel c), HDL cholesterol (mg/dl, panel d) and triglyceride levels (mg/dl, panel e) over a 12-week follow-up period after the initiation of rosuvastatin. Numeric values refer to medians.

\section{Clinical profile of responders}

The Table 2 shows the baseline clinical characteristics of "responders" - that is, patients who reached a serum LDL-c $<100 \mathrm{mg} / \mathrm{dl}$ - and "nonresponders" - that is, patients who did not. Statistically significant differences were found with regard to the time elapsed since HT, which was shorter in the former group (median 2.6 versus 8.5 years, $P=0.035$ ), and baseline serum levels of TC (median 222 versus $259 \mathrm{mg} / \mathrm{dl}$, $P=0.01$ ) and LDL-c (median 155 versus $130 \mathrm{mg} / \mathrm{dl}, P=0.01$ ), which were higher in the latter. Before the start of rosuvastatin therapy, most of "nonresponders" were being treated with atorvastatin $(62 \%)$, while most of "responders" were being treated with pravastatin (71\%). Equivalent daily doses of previous statin therapy were significantly higher among "nonresponders" than among "responders" (median 40 versus $20 \mathrm{mg}, P=0.043)$. 
Table 2. Baseline clinical characteristics of patients who had an LDL-c $<100 \mathrm{mg} / \mathrm{dl}$ at the end of the 12-week follow-up period ("responders") in comparison with those who had not ("nonresponders")

\begin{tabular}{|c|c|c|c|}
\hline & Responders & Nonresponders & $P$ \\
\hline Age (years), median (IQR) & 59.7 & 60.1() & 0.263 \\
\hline Female $(n, \%)$ & $2(12 \%)$ & $2(15 \%)$ & 0.773 \\
\hline Diabetes mellitus & $4(23 \%)$ & $2(15 \%)$ & 0.672 \\
\hline Hypertension & $11(65 \%)$ & $9(69 \%)$ & 0.794 \\
\hline \multicolumn{4}{|l|}{ Immunosuppressive therapy } \\
\hline Cyclosporine & $3(18 \%)$ & $6(46 \%)$ & 0.091 \\
\hline Tacrolimus & $11(65 \%)$ & $4(31 \%)$ & 0.065 \\
\hline Sirolimus or Everolimus & $6(35 \%)$ & $7(54 \%)$ & 0.31 \\
\hline Mycophenolate mofetil & $14(82 \%)$ & $7(58 \%)$ & 0.091 \\
\hline Prednisone & $17(100 \%)$ & $13(100 \%)$ & 1 \\
\hline \multicolumn{4}{|l|}{ Current treatment with statins } \\
\hline Pravastatin & $12(71 \%)$ & $2(15 \%)$ & \multirow{4}{*}{0.018} \\
\hline Atorvastatin & $3(18 \%)$ & $8(62 \%)$ & \\
\hline Fluvastatin & 0 & $1(3 \%)$ & \\
\hline None & $2(12 \%)$ & $2(2 \%)$ & \\
\hline Pravastatin equivalent daily dose (mg), median (IQR) & $20(20-20)$ & $40(10-70)$ & 0.043 \\
\hline Prior statin toxicity & $2(12 \%)$ & $2(15 \%)$ & 0.773 \\
\hline Hepatotoxicity & $2(12 \%)$ & $1(8 \%)$ & 0.713 \\
\hline Muscle toxicity & 0 & $1(8 \%)$ & 0.433 \\
\hline Body mass index (kg/m2), median (IQR) & $27.8(24.3-33.3)$ & $27.1(26.4-29.4)$ & 1 \\
\hline Time since HT (years), median (IQR) & $2.6(0.9-5.6)$ & $8.5(2-12.5)$ & 0.035 \\
\hline \multicolumn{4}{|l|}{ Baseline laboratory parameters, median (IQR) } \\
\hline Total cholesterol (mg/dl) & $222(203-244)$ & $259(239-305)$ & $<0.001$ \\
\hline LDL cholesterol (mg/dl) & $130(115-143)$ & $155(142-207)$ & $<0.001$ \\
\hline HDL cholesterol (mg/dl) & $57(42-70)$ & $55(44-69)$ & 0.65 \\
\hline Triglycerides (mg/dl) & $156(108-239)$ & $167(124-255)$ & 0.837 \\
\hline Creatinine (mg/dl) & $1.4(1.2-1.8)$ & $1.2(1.1-1.4)$ & 0.186 \\
\hline Total bilirubin (mg/dl) & $0.6(0.4-0.9)$ & $0.6(0.5-0.9)$ & 0.805 \\
\hline GOT (IU/l) & $20(19-26)$ & $33(19-43)$ & 0.123 \\
\hline GPT (IU/l) & $24(18-29)$ & $29(17-45)$ & 0.245 \\
\hline CPK (IU/l) & $82(50-123)$ & $143(57-278)$ & 0.079 \\
\hline
\end{tabular}

CPK, creatine phosphokinase; GOT, glutamate oxaloacetate oxalate transaminase; GPT, glutamate pyruvate transaminase, IQR, interquartile range; HT, heart transplantation.

There was a trend to a higher use of tacrolimus and a lower use of cyclosporine A among "responders," but this difference did not reach statistical significance. No significant differences between both groups were observed with regard to the use of mycophenolate mophetil or mTOR inhibitors.

\section{Long-term follow-up}

Beyond the end of the predefined 12-week follow-up period, 27 (90\%) patients continued on rosuvastatin therapy by clinical decision. At this moment, drug discontinuation was due to side effects in two cases, as detailed in the specific chapter of Adverse Reactions (page 10).

Treatment was maintained for a median time of 1772 days (IQR 1107-1877). Twenty-three patients were treated exclusively with rosuvastatin, and 4 patients also received ezetimibe in combination. At the last clinical visit, median serum levels of TC, LDL-c, HDL-c, and triglycerides were, respectively, 179 mg/dl (IQR: 142-215), 97 mg/dl (IQR: 72-114), 49 mg/dl (IQR: 41-65), and 154 mg/dl (IQR: 87- 
215), and median TC/HDL-c ratio was 3.3 (IQR: 2.9-4). No variation of serum lipid levels from the end of the predefined 12-week follow-up period to the end of long-term follow-up was statistically significant $(P>0.05$ for all comparisons). The proportion of patients who reached the secondary therapeutic goals for TC $(<200 \mathrm{mg} / \mathrm{dl})$, LDL-c $(<100 \mathrm{mg} / \mathrm{dl})$, triglycerides $(<150 \mathrm{mg} / \mathrm{dl})$, and TC/HDL-c ratio $(<4)$ at the end of long-term follow-up were $67 \%, 63 \%, 48 \%$, and $73 \%$, respectively. No coronary or cerebrovascular event occurred over long-term follow-up in studied patients.

\section{Adverse reactions}

No significant changes in the distribution of serum levels of GOT, GPT, bilirubin, or CPK in the study population were observed during the predefined 12-week follow-up period after the initiation of rosuvastatin (Fig. 2).
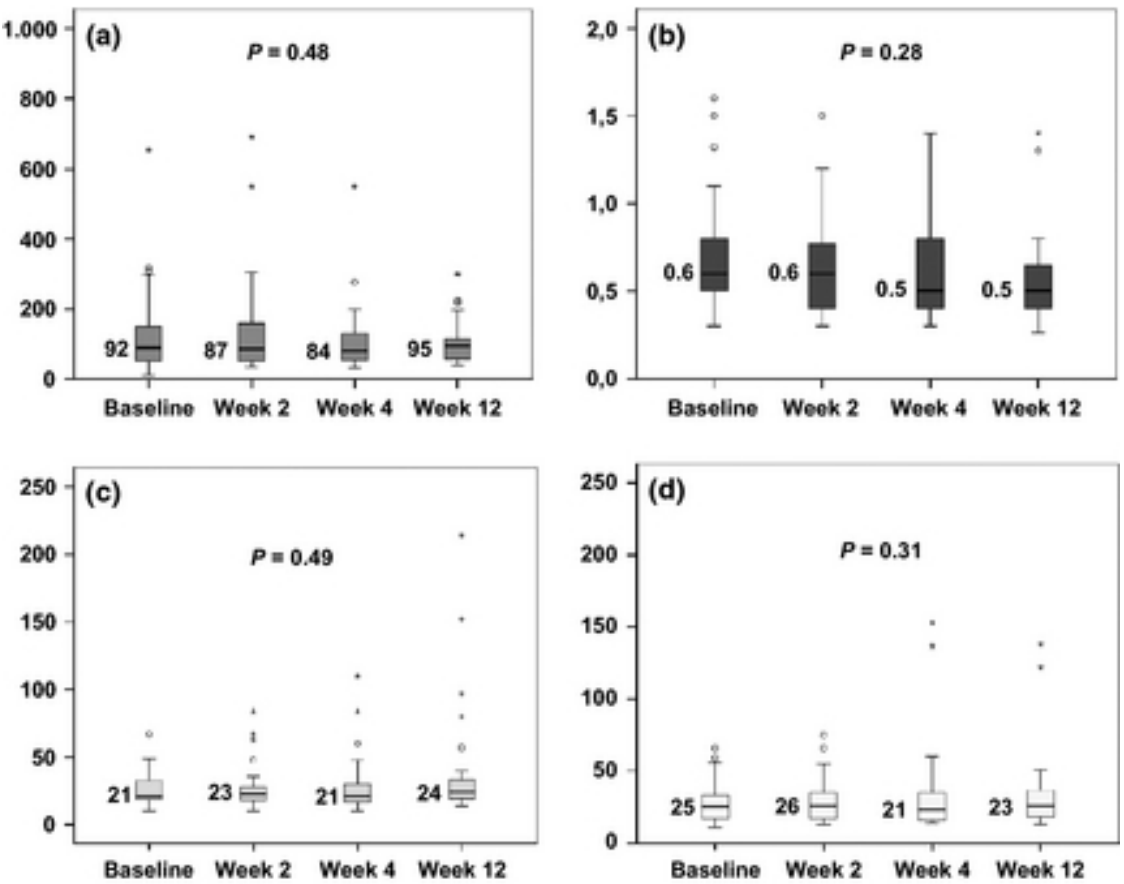

Figure 2. Evolution of serum levels of CPK (IU/liter, panel a), total bilirubin (mg/dl, panel b), GOT (UI/liter, panel c) and GPT (IU/l, panel d) during a 12-week follow-up period after the initiation of rosuvastatin. Numeric values refer to medians. ${ }^{\circ}$,outliers, *,extreme values.

Over the whole follow-up of the study, rosuvastatin was suspended in $2(6.7 \%)$ patients due to muscle toxicity - 1 case of rhabdomyolysis and 1 case of myalgia without CPK elevation-and in 1 (3.3\%) patient due to liver toxicity - asymptomatic elevation of transaminases, so as resulting in an incidence rate of adverse reactions leading to drug suspension of 2.78 episodes per 100 patient-years-1.85 episodes of muscle toxicity per 100 patient-years and 0.93 episodes of liver toxicity per 100 patient-years. The onset of these three cases of toxicity was 21,25 , and 318 days after the initiation of rosuvastatin therapy, respectively.

Both patients with muscular toxicity received concomitant therapy with cyclosporine and everolimus when rosuvastatin was initiated. At the time of the side effect, serum levels of cyclosporine were within the normal range $(79.9$ and $60.1 \mathrm{ng} / \mathrm{ml})$, but one patient presented slight everolimus overdose $(8.5 \mathrm{ng} / \mathrm{ml})$. The patient with liver toxicity was being treated with mycophenolate and tacrolimus. Both this patient and the patient with myalgia had already presented similar adverse reactions to other statins previously. All adverse events resolved within a few days after drug withdrawal without requiring any other specific therapeutic intervention and without permanent sequelae. 


\section{Discussion}

The results of our study suggest that rosuvastatin therapy is effective in reducing TC and LDL-c in patients with HT in which other statin regimens have failed to achieve the recommended therapeutic goals. Moreover, this benefit is not accompanied by an undesirable incidence of adverse effects.

Practice guidelines recommend chronic statin therapy in all patients with HT [3]. In this population, the rationale for statin therapy goes beyond their lipid-lowering action or cardiovascular risk reduction, as they are also thought to act as immune modulators [14]. Two randomized clinical trials have studied the potential clinical benefit of pravastatin [7] and simvastatin in patients with HT [8], showing a significant reduction in the incidence of acute rejection and coronary allograft vasculopathy and an improvement in long-term survival compared to the placebo group. This occurs in addition to the expected reduction in total serum cholesterol and LDL cholesterol.

In the general population with hypercholesterolemia, treatment with $10 \mathrm{mg} /$ day of rosuvastatin leads to an average relative reduction in serum LDL-c levels of $46 \%$ [10], which is comparable to that obtained with high doses of atorvastatin $(40 \mathrm{mg} /$ day $)$ or simvastatin $(80 \mathrm{mg} / \mathrm{day})$. In our study, the mean relative reduction in LDL-c was significantly lower (19\%), similar to what was observed in a previous study in patients with HT [12]. Although we cannot rule out the hypothesis of a poor response to the drug in patients with HT compared to the general population, our view is that this discrepancy may be explained by a selection bias resulting from the inclusion criteria of the study, as all patients included had a plasma LDL-c level $>100 \mathrm{mg} / \mathrm{dl}$ despite previous statin treatment, with a median daily dose equivalent to $36 \mathrm{mg}$ of pravastatin. The frequent use of proliferation signal inhibitors is another important factor to take into account, given that these drugs may induce dyslipidemia difficult to control despite treatment with lipidlowering agents [15]. In our series, as it has been shown in other studies [12, 16], we observed no significant effect of rosuvastatin on serum HDL cholesterol levels. The slight reduction in plasma triglyceride levels observed in our study showed no statistical significance, contrarily to what was reported by other authors $[12,16]$.

In our study, 57\% patients treated with rosuvastatin achieved a LDL-c level <100 mg/dl. This proportion is slightly lower than that observed by Samman et al. [12]. Nonetheless, we feel that it is a relevant reduction, taking into account that all patients presented with levels above this threshold before the start of the study, due to insufficient response or intolerance to other statins. By analogy to the recommendations of lipid control in patients with high cardiovascular risk [17], some authors argue that an LDL-c level $<100 \mathrm{mg} / \mathrm{dl}$ might be a reasonable target to pursue in most patients with HT [18], given the high prevalence of risk factors in this population. However, the risk-benefit ratio of this strategy must be individualized, given the known increased susceptibility to drug toxicity of HT recipients [9, 10].

A major strength of our investigation in comparison with the previous study [12] of rosuvastatin in HT recipients is the availability of long-term results. Because of clinical decision, $90 \%$ of patients continued on rosuvastatin therapy after the pre-defined 12-week follow-up period of the study, with a median period of time on treatment of $\sim 3.5$ years. Serum levels of LDL-c and TC at the last clinical visit remained unchanged in comparison with the end of the predefined 12-week follow-up period, so as supporting the sustained efficacy of rosuvastatin therapy in the HT population in the long term. The proportion of patients matching the therapeutic goal of a serum LDL-c $<100 \mathrm{mg} / \mathrm{dl}$ in the long term was $63 \%$, and this proportion was even slightly higher for other secondary therapeutic goals of lipid profile as a TC $<200 \mathrm{mg} / \mathrm{dl}(67 \%)$ or a TC/HDL-c ratio <4 (73\%). Interestingly, no cerebrovascular or coronary event occurred among studied subjects over long-term follow-up. Despite it is a good result, the study was not designed, nor powered, to detect clinical events, so no conclusion may be extracted about the efficacy of the drug to prevent cardiovascular complications in the HT population.

Trying to identify baseline clinical characteristics that might be associated with a higher probability of experiencing a significant therapeutic response to rosuvastatin therapy, we conducted an exploratory, descriptive analysis comparing the clinical profile of patients who reached a LDL-c $<100 \mathrm{mg} / \mathrm{dl}$ after treatment onset ("responders") and patients who did not ("nonresponders"). A shorter time since HT to treatment onset, a lower baseline LDL-c and a lower intensity of previously failed statin therapy seemed to be the unique clinical characteristics that precluded a higher probability for a patient to become a "responder." This conclusion, however, must be taken with caution, as the limited size of the study sample prevented us to conduct the required multivariable adjustment. 
In the general population, adverse reactions to statins are infrequent and dose-related and usually disappear after drug withdrawal [19]. The most common adverse event following statin therapy is muscle pain, which can occur in up to $5 \%$ of patients, although the incidence of severe myopathy is lower than $0.2 \%$ [20, 21]. Liver toxicity is also infrequent, as it occurs in less than $2 \%$ of patients treated with statins, usually in an asymptomatic manner, and disappears after discontinuation of treatment [22]. In an exhaustive review [23] of more than 16000 patients enrolled in clinical trials comparing rosuvastatin vs. other statins, the reported cumulative incidence of liver toxicity and muscle toxicity was, respectively, $0.2 \%$ and $0.3 \%$. The hydrophilic nature of rosuvastatin and its low affinity for the hepatic cytochrome P450 are the proposed reasons for the apparently good safety profile of this drug [24].

In patients with HT, there is an increased risk of statin-related muscle and liver toxicity [9, 10], which is attributed to drug-drug interactions with immunosuppressive agents [25]. In the case of rosuvastatin, the risk of interaction is especially high with cyclosporine, which induces a competitive inhibition of the liver transporter organic anion transporting polypeptide $C$ [26]. This protein is essential for the degradation of drug; because of this, concomitant treatment with rosuvastatin and cyclosporine usually results in an increased plasmatic concentration of the lipid-lowering agent.

Over a $\sim 3.5$-year follow-up, we detected two cases $(6.7 \%)$ of muscle toxicity and one case $(3.3 \%)$ of liver toxicity associated with rosuvastatin, which were not life-threatening and reversed without sequelae after drug withdrawal. Both cases of muscular toxicity appeared early ( $<30$ days) after the initiation of the drug, and the other was detected after 10 months on treatment. With variable lengths of treatment duration among studies, the reported cumulative incidence of statin-related toxicity in the HT population was around $8 \%$ with atorvastatin [27], 13\% with fluvastatin [28] and simvastatin [29], and significantly lower with pravastatin [29]. In a previous study by our own group, we observed an overall cumulative incidence of statin toxicity of $12 \%$ [9] in HT patients, being obesity the only risk factor independently associated with this complication. Samman et al. [12] detected 1 case of myalgia and two cases of asymptomatic mild CPK elevation in their cohort of 20 HT patients treated with rosuvastatin for a 6-week period.

Given that the cumulative incidence of adverse reactions to rosuvastatin in our study was at least not higher than reported in studies with other statins in the HT population, that our series included a significant proportion (13\%) of patients with previous adverse reactions to other statins - indeed, two of the three cases of toxicity occurred in patients with this antecedent - and that all adverse effects were reversible after drug withdrawal, we conclude that rosuvastatin is a reasonably safe therapeutic alternative in HT patients. Notwithstanding this, careful monitoring and a high level of clinical suspicion about potential adverse effects should be maintained when prescribing rosuvastatin — or other statins — to a HT recipient.

In conclusion, our study supports rosuvastatin as a good alternative for the treatment of uncontrolled hypercholesterolemia in HT recipients with a poor previous response to other statins. Rosuvastatin showed to be effective to reduce serum levels of TC and LDL-c, allowing that more than a half of such patients reach the recommended therapeutic goals for lipid profile. This result was achieved at the expense of rate of adverse reactions clearly higher than that observed in patients from the general population, but similar to observed with other statins in the HT population. Larger studies are warranted to elucidate the potential benefit of rosuvastatin in terms of cardiovascular mortality and survival in HT patients.

\section{Authorship}

GB-C, EB-C and MGC-L: provided conception and design, data acquisition and data analysis and interpretation. They wrote, reviewed and edited the manuscript. RM-R, MJP-M, AB-R and JMV-R: reviewed the manuscript critically. CN-L, PB-C and ZG-C: contributed to provide conception and design, data acquisition and data analysis and interpretation.

\section{Funding}

None. 


\section{Acknowledgements}

The authors are indebted to Alberto L. García-Basteiro, MD, MSc for linguistic advice.

\section{References}

1. Brugts J, Yetgin T, Hoeks S, et al. The benefits of statins in people without established cardiovascular disease but with cardiovascular risk factors: meta-analysis of randomised controlled trials. BMJ 2009; 338: b2376.

2. Josan K, Majundar S, McAlister F. The efficacy and safety of intensive statin therapy: a meta-analysis of randomized trials. CMAJ 2008; 178: 576.

3. Costanzo MR, Dipchand A, Starling R, et al. The International Society of Heart and Lung Transplantation Guidelines for the care of heart transplant recipients. J Heart Lung Transplant 2010; 29: 914.

4. Kobashigawa JA, Kasiske BL. Hyperlipidemia in solid organ transplantation. Transplantation 1997; 63: 331.

5. Hricik DE, Mayes JT, Schulak JA. Independent effects of cyclosporine and prednisone on post-transplant hypercholesterolemia. Am J Kidney Dis 1991; 18: 353.

6. Weis M, Von Scheidt W. Cardiac allograft vasculopathy: a review. Circulation 1997; 96: 2069.

7. Wenke K, Meiser B, Thiery J, et al. Simvastatin reduces graft vessel disease and mortality after heart transplantation: a four-year randomized trial. Circulation 1997; 96: 1398.

8. Kobashigawa J, Katznelson S, Laks H, et al. Pravastatin on outcomes after cardiac transplantation. N Eng J Med 1995; 333: 621 .

9. Marzoa-Rivas R, Crespo-Leiro M, Paniagua-Martín MJ, et al. Safety of statins when response is carefully monitored: a study of 336 heart recipients. Transplant Proc 2005; 37: 4071.

10. Grigioni F, Carigi S, Potena L, et al. Long-term safety and effectiveness of statins for heart transplant recipients in routine clinical practice. Transplant Proc 2006; 38: 1507.

11. Jones PH, Davidson MH, Stein EA, et al. Comparison of the efficacy and safety of rosuvastatin versus atorvastatin, simvastatin, and pravastatin across doses (STELLAR* Trial). Am J Cardiol 2003; 92: 152.

12. Samman A, Imai C, Straatman L, et al. Safety and efficacy of rosuvastatin therapy for the prevention of hyperlipidemia in adult cardiac transplant recipients. J Heart Lung Transplant 2005; 24: 1008.

13. Mancini GB, Baker S, Bergeron J, et al. Diagnosis, prevention, and management of statin adverse effects and intolerance: Proceedings of a Canadian Working Group Consensus Conference. Can J Cardiol 2001; 27 : 635.

14. Kwak B, Mulhaupt F, Myit S, et al. Statins as a newly recognized type of immunomodulator. Nat Med 2000; 6: 1399.

15. Aliabadi AZ, Mahr S, Dunkler D, et al. Safety and efficacy of statin therapy in patients switched from cyclosporine a to sirolimus after cardiac transplantation. Transplantation 2008; 86: 1771

16. Rader DJ, Davidson MH, Caplan RJ, et al. Lipid and apolipoprotein ratios: association with coronary artery disease and effects of rosuvastatin compared with atorvastatin, pravastatin and simvastatin. Am J Cardiol 2003; 91: 20C.

17. Grundy SM, Cleeman JI, Merz CN, et al. Implications of recent clinical trials for the National Cholesterol Education Program Adult Treatment Panel III guidelines. Circulation 2004; 110: 227.

18. Massy ZA. Hyperlipidemia and cardiovascular disease after organ transplantation. Transplantation $2001 ; 72(6$ Suppl.): S13.

19. Chatzizisis YS, Koskinas KC, Misirli G, et al. Risk factors and drug interactions predisposing to statin-induced myopathy: implications for risk assessment, prevention and treatment. Drug Saf 2010; 33: 171.

20. Thompson PD, Clarkson P, Karas RH. Statin-associated myopathy. JAMA 2003; 289: 1681.

21. Ballantyne CM, Corsini A, Davidson MH, et al. Risk for myopathy with statin therapy in high-risk patients. Arch Intern Med 2003; 163: 553.

22. De Denus S, Spinler SA, Miller K, et al. Statins and liver toxicity: a meta-analysis. Pharmacotherapy 2004; 24: 584.

23. Shepherd J, Vidt D, Miller E, et al. Safety of rosuvastatin: update on 16,876 rosuvastatin-treated patients in a multinational clinical trial program. Cardiology 2007; 107: 433.

24. Olsson AG, McTaggart F, Raza A. Rosuvastatin: a highly effective new HMG-CoA reductase inhibitor. Cardiovasc Drug Rev 2002; 20: 303.

25. Anglicheau D, Legendre C, Beaune $\mathrm{P}$, et al. Cytochrome $\mathrm{P} 450$ 3A polymorphisms and immunosuppressive drugs: an update. Pharmacogenomics $2007 \mathrm{Jul} ; 8$ : 835.

26. Simonson SG, Raza A, Martin PD, et al. Rosuvastatin pharmacokinetics in heart transplant recipients administered an antirejection regimen including cyclosporine. Clin Pharmacol Ther 2004; 76: 167.

27. Patel DN, Pagani FD, Koelling TM, et al. Safety and efficacy of atorvastatin in heart transplant recipients. $J$ Heart Lung Transplant 2002; 21: 204.

28. Potena L, Grigioni F, Ortolani P, et al. Safety and efficacy of early aggressive versus cholesterol-driven lipidlowering strategies in heart transplantation: a pilot, randomized, intravascular ultrasound study. J Heart Lung Transplant 2011; 30: 1305.

29. Keogh A, Macdonald P, Kaan A, et al. Efficacy and safety of pravastatin vs simvastatin after cardiac transplantation. J Heart Lung Transplant 2000; 19: 529. 